\title{
Monopole charge density wave states in Weyl semimetals
}

\author{
Eric Bobrow ${ }^{\circ}$, Canon Sun, and Yi Li \\ Department of Physics and Astronomy, The Johns Hopkins University, Baltimore, Maryland 21218, USA
}

(Received 14 January 2019; revised manuscript received 1 November 2019; accepted 19 February 2020; published 26 March 2020)

\begin{abstract}
We study a class of topological charge density wave states exhibiting monopole harmonic symmetries. The density wave ordering is equivalent to pairing in the particle-hole channel due to Fermi surface nesting under interactions. When electron and hole Fermi surfaces carry different Chern numbers, the particle-hole pairing exhibits a nontrivial Berry phase inherited from band-structure topology independent of the concrete density wave ordering mechanism. The associated density wave gap functions become nodal, and the net nodal vorticity is determined by the monopole charge of the pairing Berry phase. The gap function nodes become zero-energy Weyl nodes of the bulk spectra of quasiparticle excitations. These states can occur in doped Weyl semimetals with nested electron and hole Fermi surfaces enclosing Weyl nodes of the same chirality in the weak-coupling regime. Topologically nontrivial low-energy Fermi arc surface states appear in the density wave ordering state as a consequence of the emergent zero-energy Weyl nodes.
\end{abstract}

DOI: 10.1103/PhysRevResearch.2.012078

Introduction. Charge density wave (CDW) ordering, the spontaneous ordering of electron density or bond strength, is an important phenomenon in correlated electron systems [1,2]. The broken translational symmetry of CDW ordering often arises from a Peierls instability, which is driven by electron-phonon interactions between nested Fermi surfaces that lead to the softening of phonon modes and accompanying periodic lattice distortions [3]. Topological electron excitations can exist at defects in the CDW order, such as half-fermion modes localized around the domain walls of the Peierls distortion in the one-dimensional polyacetylene chain $[2,4]$. The CDW instability may also be driven by electron-electron interactions, as studied in the context of high- $T_{c}$ cuprates [5-7]. Analogous to unconventional superconductivity, CDW order may also possess unconventional symmetries, forming a nontrivial representation of the lattice symmetry group. For example, CDW order with a $d$-wave form factor was proposed to compete and coexist with superconductivity $[5,6]$.

Study of the Berry phase of Bloch wave states in lattice systems has led to the discovery of a plethora of topological states, such as quantum anomalous Hall insulators [8,9] and topological insulators [10-13]. Furthermore, the discovery of Weyl semimetals [14-48] has stimulated the study of topological phases in semi-metallic systems. As in quantum anomalous Hall insulators where a Chern number structure arises from quantized Berry flux over the two-dimensional Brillouin zone, in three-dimensional semimetals the Fermi surfaces have a Chern number structure due to the Weyl points acting as sources or sinks of Berry flux.

Published by the American Physical Society under the terms of the Creative Commons Attribution 4.0 International license. Further distribution of this work must maintain attribution to the author(s) and the published article's title, journal citation, and DOI.
After doping, magnetic Weyl semimetals can host topological monopole harmonic superconductivity, a class of topological states. As opposed to typical unconventional superconductors, such as $d$-wave high- $T_{c}$ cuprates, and $p$-wave superfluid ${ }^{3} \mathrm{He}$, in monopole harmonic superconductors the gap function $\Delta(k)$ cannot be described by spherical harmonics and their lattice counterparts [48]. Instead, these systems carry "pairing monopole charge," a generalization of Berry phase from single-particle states to a two-particle order parameter. When the pairing occurs between two Fermi surfaces with opposite Chern numbers, which can be the case when the enclosed Weyl points have opposite chiralities, Cooper pairs acquire nontrivial Berry phase structure. As a result, the gap function cannot be well defined over the entire Fermi surface. Consequently, the Fermi surface becomes nodal with total vorticity determined by the pairing monopole charge associated with the two-particle Berry phase.

In this Rapid Communication, we study nontrivial Berry phase structure for a class of order parameter gap functions lying in the particle-hole channel. As an example that can be realized in a doped Weyl semimetal, CDW ordering will be considered. When two nested Fermi surfaces, one electronlike and one holelike, carry different Chern numbers, the CDW order formed between these two Fermi surfaces inherits nontrivial band-structure topology that can be seen in its gap function $\rho(\mathbf{k})$. As with the gap function of monopole harmonic superconductivity, $\rho(\mathbf{k})$ cannot be globally well defined in momentum space and becomes nodal. The nontrivial Berry flux enforces a nonzero total vorticity of $\rho(\mathbf{k})$ determined by the difference in Chern number between the two nested Fermi surfaces, independent of the concrete mechanism for CDW ordering. The nodes of the CDW gap function emerge as new Weyl nodes in the low-energy quasiparticle spectra that are distinct from, but have chiralities determined by, the original band-structure Weyl points.

Gap function Berry flux and nodes for CDW ordering. We begin with a minimal description of a pair of electronlike and 
holelike Fermi surfaces, which carry opposite Chern numbers and are well nested. Such Fermi surfaces can be realized in a three-dimensional (3D) Weyl semimetal system around two Weyl points of the same chirality. Consider two Weyl points of positive chirality located at $\mathbf{K}_{e}^{+}$and $\mathbf{K}_{h}^{+}$with energies $-E_{0}$ and $E_{0}$ respectively and Fermi energy at $\mu=0$. A holelike Fermi surface denoted $\mathrm{FS}_{h, C}$ is centered around $\mathbf{K}_{h}^{+}$with Chern number $C$ or, equivalently, monopole charge $q=\frac{1}{2} C$. Similarly, an electronlike Fermi surface denoted $\mathrm{FS}_{e,-C}$ is centered around $\mathbf{K}_{e}^{+}$with Chern number $-C$. An example system with this Fermi-surface structure is considered in Eq. (3) below.

Since $\mathrm{FS}_{h, C}$ and $\mathrm{FS}_{e,-C}$ are well nested, they favor a CDW instability, inter-Fermi-surface particle-hole pairing, under repulsive interactions. The two-particle CDW order parameter exhibits a nontrivial Berry flux quantization, which can be seen as follows. After projecting to the low-energy Fermi surfaces, the electron creation operators on $\mathrm{FS}_{h, C}$ and $\mathrm{FS}_{e,-C}$ can be defined as $\alpha_{ \pm}^{\dagger}(\mathbf{p})=\sum_{a=\uparrow, \downarrow} \xi_{ \pm, a}(\mathbf{p}) c_{a}^{\dagger}\left(\mathbf{K}_{h(e)}^{+}+\mathbf{p}\right)$, where $\mathbf{p}$ is the momentum relative to the Weyl node at $\mathbf{K}_{h(e)}^{+}, a$ refers to the spin or pseudospin degrees of freedom, and $\xi_{ \pm}(\mathbf{p})$ is the spinor eigenfunction carrying monopole charge $\pm q$. Here $\mathbf{p}$ lies on the surface $S$ that results from shifting $\mathrm{FS}_{e,-C}$ by $-\mathbf{K}_{e}^{+}$ towards the origin. We define the particle-hole channel pairing operator

$$
P_{-+}(\mathbf{p})=\alpha_{-}^{\dagger}(\mathbf{p}) \alpha_{+}(\mathbf{p}),
$$

which creates an electron on $\mathrm{FS}_{e,-C}$ and a hole on $\mathrm{FS}_{h, C}$. The single-particle Berry connection is $\mathbf{A}_{ \pm}(\mathbf{p})=$ $\sum_{a} i \xi_{ \pm, a}^{*}(\mathbf{p}) \nabla_{\mathbf{p}} \xi_{ \pm, a}(\mathbf{p})$, and the Berry flux penetrating $\mathrm{FS}_{ \pm}$ is given by $\oiint_{S} d \mathbf{p} \cdot \nabla_{\mathbf{p}} \times \mathbf{A}_{ \pm}(\mathbf{p})= \pm 4 \pi q$. It can be shown that the pairing Berry connection associated with $P_{-+}(\mathbf{p})$ is $\mathbf{A}_{-+}(\mathbf{p})=\mathbf{A}_{-}(\mathbf{p})-\mathbf{A}_{+}(\mathbf{p})$, and the net pairing Berry flux through $S$ is $\oiint_{S} d \mathbf{p} \cdot \nabla_{\mathbf{p}} \times \mathbf{A}_{-+}(\mathbf{p})=4 \pi q_{\mathrm{CDW}}$, where $q_{\mathrm{CDW}}=-2 q$.

The nonzero Berry flux through $S$ leads to a nontrivial vortex structure for the CDW gap function. The CDW interaction Hamiltonian after mean-field decomposition is expressed as

$$
H_{\rho}=\sum_{\mathbf{p}} \rho_{-+}(\mathbf{p}) P_{-+}(\mathbf{p})+\rho_{+-}(\mathbf{p}) P_{+-}(\mathbf{p}),
$$

where $P_{+-}=P_{-+}^{\dagger}$. The gap function $\rho_{-+}$is conjugate to the CDW operator $P_{-+}(\mathbf{p})$, and $\rho_{+-}=\rho_{-+}^{*}$. Because of the nontrivial gauge field $\mathbf{A}_{-+}, \rho_{-+}(\mathbf{p})$ cannot be globally well defined on $S$. This follows from examining the gauge invariant circulation field $\mathbf{v}_{-+}(\mathbf{p})=\nabla \phi_{-+}(\mathbf{p})-\mathbf{A}_{-+}(\mathbf{p})$, where $\phi_{-+}$is the phase of $\rho_{-+} \cdot \mathbf{v}_{-+}$is well defined except at the nodes of $\rho_{-+}(\mathbf{p})$, and each node has an integer-valued vorticity $g_{i}=\frac{1}{2 \pi} \oint_{C_{i}} d \mathbf{p} \cdot \mathbf{v}_{-+}(\mathbf{p}) . C_{i}$ is an infinitesimal loop around the node $\mathbf{p}_{i}$, with the positive loop direction defined with respect to the local normal vector. The total vorticity of $\rho_{-+}$over $S$ is $\sum_{i} g_{i}=2 q_{\mathrm{CDW}}$, where the sum is over all the nodes on $S$. As a result, the enclosure of a nonzero net monopole charge gives rise to nodes of $\rho_{-+}(\mathbf{p})$ on $S$. The nontrivial nodal structure necessitates the use of monopole harmonic functions [49], as opposed to the usual spherical harmonics, to describe the order parameter.

Doped Weyl semimetal with nested Fermi surfaces. To demonstrate the above topological nodal structure, we employ

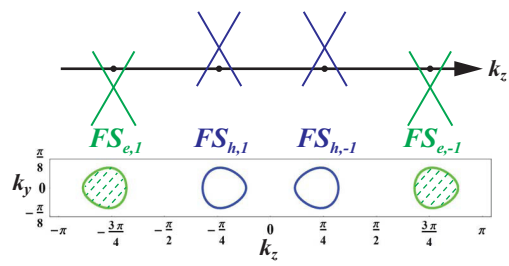

(a)

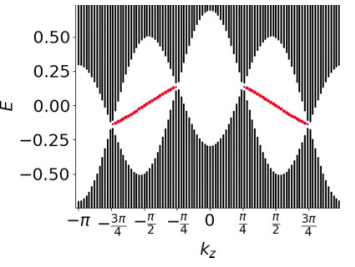

(b)
FIG. 1. (a) The four Fermi surfaces of the band Hamiltonian in Eq. (4) with $V_{0} / t=0.49$. They are divided into two well-nested pairs: $\mathrm{FS}_{h, 1}$ and $\mathrm{FS}_{e,-1}$ which enclose the Weyl points $\mathbf{K}_{h, e}^{+}$with positive chirality, and $\mathrm{FS}_{h,-1}$ and $\mathrm{FS}_{e, 1}$, which enclose the Weyl points $\mathbf{K}_{e, h}^{-}$with negative chirality. (b) Energy spectrum as a function of $k_{z}$ in the absence of the CDW ordering and $V_{0} / t=0.2$. The surface states localized on the $y=0$ boundary are plotted in red connecting $\mathbf{K}_{e}^{-}$and $\mathbf{K}_{h}^{+}$and $\mathbf{K}_{h}^{-}$and $\mathbf{K}_{e}^{+}$.

the band Hamiltonian

$$
H=\sum_{\substack{\mathbf{k}, a, b}} c_{a}^{\dagger}(\mathbf{k})[h(\mathbf{k})+V(\mathbf{k})]_{a b} c_{b}(\mathbf{k})+H_{\rho},
$$

where $a, b$ refers to the pseudospin degree of freedom, typically realized by $A$ and $B$ sublattices; $H_{\rho}$ is the mean-field Hamiltonian for CDW ordering specified below; and we assume the chemical potential $\mu=0$. The matrix kernel $h(\mathbf{k})$ of the band Hamiltonian is

$$
\begin{aligned}
h(\mathbf{k})= & t_{z}\left(2-\cos k_{x}-\cos k_{y}-\gamma+\cos ^{2} k_{z}\right) \tau_{z} \\
& +t_{x} \sin k_{x} \tau_{x}+t_{y} \sin k_{y} \tau_{y},
\end{aligned}
$$

with Pauli matrices $\tau_{x, y, z}$ defined in the $A, B$ basis and pseudospin-dependent hopping amplitudes $t_{x, y, z}$. Here $\gamma=$ $1 / 2$ controls the location of the Weyl points along $\mathbf{k}_{z}$. For simplicity, we choose $t_{x, y, z}=t$ in this Rapid Communication. The corresponding lattice model giving rise to $h(\mathbf{k})$ is presented in Supplemental Material (SM) I [50]. The momentumdependent potential $V(\mathbf{k})$ takes the form

$$
V(\mathbf{k})=V_{0} \cos k_{z} I,
$$

with $I$ the $2 \times 2$ identity matrix. $V_{0}$ plays a role similar to a chemical potential by controlling the size of the Fermi surface.

Without loss of generality, we assume $V_{0}>0$. This model possesses four Weyl points, all located on the $k_{z}$ axis, at $\mathbf{K}_{e}^{-}=\left(0,0,-\frac{3 \pi}{4}\right), \mathbf{K}_{h}^{+}=\left(0,0,-\frac{\pi}{4}\right), \mathbf{K}_{h}^{-}=-\mathbf{K}_{h}^{+}$, and $\mathbf{K}_{e}^{+}=-\mathbf{K}_{e}^{-}$, where the upper indices \pm refer to the chiralities of the Weyl points and the lower indices $e$ and $h$ refer to whether the Fermi surface associated with the Weyl point is electronlike or holelike. The potential $V(\mathbf{k})$ shifts the points $\mathbf{K}_{h}^{-}$and $\mathbf{K}_{h}^{+}$up in energy, forming the respective holelike Fermi surfaces $\mathrm{FS}_{h, 1}$ and $\mathrm{FS}_{h,-1}$, as shown in Fig. 1(a). Similarly, the points $\mathbf{K}_{e}^{+}$and $\mathbf{K}_{e}^{-}$are shifted down in energy, forming the electron pockets $\mathrm{FS}_{e,-1}$ and $\mathrm{FS}_{e,+1}$. This model is a modification of the models in Refs. [17,51] to allow four Weyl points with nesting. The electron and hole Fermi pockets enclosing the Weyl points with the same chirality are nested with the commensurate wave vector $\mathbf{Q}=(0,0, \pi)$. This nesting condition is satisfied so that portions of the Fermi surface separated by $\mathbf{Q}$ have the same shape. Under an open boundary condition along the $y$ direction and periodic 
boundary conditions along $x$ and $z$, the energy spectrum in the absence of the CDW ordering is plotted in Fig. 1(b) as a function of $k_{z}$ along the $k_{x}=0$ cut. The surface Fermi arc states are shown in red.

CDW ordering is imposed through the mean-field Hamiltonian

$$
H_{\rho}=\sum_{\substack{\mathbf{k}, a, b=A, B}} c_{a}^{\dagger}(\mathbf{k}+\mathbf{Q}) \rho_{a b}(\mathbf{k}) c_{b}(\mathbf{k})+\text { H.c. },
$$

where we take $\rho(\mathbf{k})=\rho \tau_{z}$ and $\rho$ is the magnitude of the CDW ordering. $\rho(\mathbf{k})$ is diagonal in the sublattice $A$ and $B$ basis, which describes two sublattices with different charge densities. Below we will see that this CDW ordering does not open a full gap over the Fermi surface but instead becomes nodal with a nontrivial vorticity.

Topological nodal CDW. We first consider the CDW gap function connecting $\mathrm{FS}_{h, 1}$ and $\mathrm{FS}_{e,-1}$ which enclose the Weyl nodes $\mathbf{K}_{h}^{+}$and $\mathbf{K}_{e}^{+}$, respectively. For small $V_{0} / t$ and $\rho / t$, the Fermi surfaces are close to the Weyl nodes and the single-particle states correspond to the helicity eigenstates satisfying $\hat{\mathbf{p}} \cdot \tau \xi_{ \pm}=\mp \xi_{ \pm}$, where $\xi_{ \pm}$corresponds to Berry flux monopole charges $q= \pm \frac{1}{2}$. Explicitly, $\xi_{ \pm}$can be represented as $\xi_{+}(\hat{\mathbf{p}})=\left(-\sin \frac{\theta_{p}}{2} e^{-i \phi_{p}}, \cos \frac{\theta_{p}}{2}\right)^{T}$ and $\xi_{-}(\hat{\mathbf{p}})=$ $\left(\cos \frac{\theta_{p}}{2}, \sin \frac{\theta_{p}}{2} e^{i \phi_{p}}\right)^{T}$, where $\theta_{p}$ and $\phi_{p}$ are the polar and azimuthal angles of $\hat{\mathbf{p}}$ and a gauge convention has been chosen. The electron creation operator for the eigenstate on the helical Fermi surface $\mathrm{FS}_{h, 1}$ is $\alpha_{+, h}^{\dagger}(\mathbf{p})=\sum_{a} \xi_{+, a}(\hat{\mathbf{p}}) c_{a}^{\dagger}\left(\mathbf{K}_{h}^{+}+\mathbf{p}\right)$, and that for $\mathrm{FS}_{e,-1}$ is $\alpha_{-, e}^{\dagger}(\mathbf{p})=\sum_{a} \xi_{-, a}(\hat{\mathbf{p}}) c_{a}^{\dagger}\left(\mathbf{K}_{e}^{+}+\mathbf{p}\right)$.

The gap function $\rho \tau_{z}$ in Eq. (6) can be projected onto the helical Fermi surfaces $\mathrm{FS}_{h, 1}$ and $\mathrm{FS}_{e,-1}$, where the projected gap function conjugate to $P_{-+}(\mathbf{p})=\alpha_{-, e}^{\dagger}(\mathbf{p}) \alpha_{+, h}(\mathbf{p})$ is $\rho_{-+}(\mathbf{p})=-\rho \sqrt{8 \pi / 3} Y_{-1,1,0}\left(\theta_{p}, \phi_{p}\right)$, in terms of monopole harmonics $Y_{q l m}$ [49]. For $\mathbf{p}$ near the north pole of $\mathrm{FS}_{e,-1}$, where $\theta_{p}=0$, the projected gap function is $\rho_{-+}^{N}(\mathbf{p})=$ $-\rho \sin \theta_{p} e^{-i \phi_{p}}$. By applying a gauge transformation, the projected gap function near the south pole, where $\theta_{p}=\pi$, can be similarly shown to be $\rho_{-+}^{S}(\mathbf{p})=-\rho \sin \theta_{p} e^{i \phi_{p}}$. The projected gap function has nodes at the poles, where $\sin \theta_{p}=0$. After taking into account the contribution of the Berry connection $\mathbf{A}_{-+}(\mathbf{p})$, the circulation field of the gap function is $\mathbf{v}_{-+}(\mathbf{p})=$ $-\cot \theta_{p} \hat{\phi}_{p}$. Integrating $\mathbf{v}$ around infinitesimal loops near the poles reveals a gap function vorticity of -1 near both, hence the total vorticity is -2 on the Fermi surface surrounding $\mathrm{FS}_{e,-1}$, consistent with $q_{\mathrm{CDW}}=-1$.

The CDW gap function nodes are actually low-energy Weyl nodes generated by interactions for the mean-field Hamiltonian. Around the nested Fermi surfaces $\mathrm{FS}_{e,-1}$ and $\mathrm{FS}_{h, 1}$, the low-energy two-band Hamiltonian is

$$
\begin{aligned}
H_{2 \text { band }}= & \sum_{\mathbf{p}} \psi^{\dagger}(\mathbf{p})\left\{(t|p|-\mu) \sigma_{z}-\rho \sin \theta_{\mathbf{p}}\left(e^{-i \phi_{p}} \sigma_{+}\right.\right. \\
& \left.\left.+e^{i \phi_{p}} \sigma_{-}\right)\right\} \psi(\mathbf{p}),
\end{aligned}
$$

where $\psi(\mathbf{p})=\left(\alpha_{-, e}(\mathbf{p}), \alpha_{+, h}(\mathbf{p})\right)^{T}$ and $\mu=-V\left(\mathbf{K}_{e}^{+}+\mathbf{p}\right)$. The interaction-induced Weyl node at the north pole, denoted $\mathbf{K}_{n}^{+}$, has positive chirality as can be shown by expanding $H_{2 \text { band }}$ about the north pole, where the helical basis is regular. The south pole, denoted $\mathbf{K}_{s}^{+}$, is the site of a singularity in the
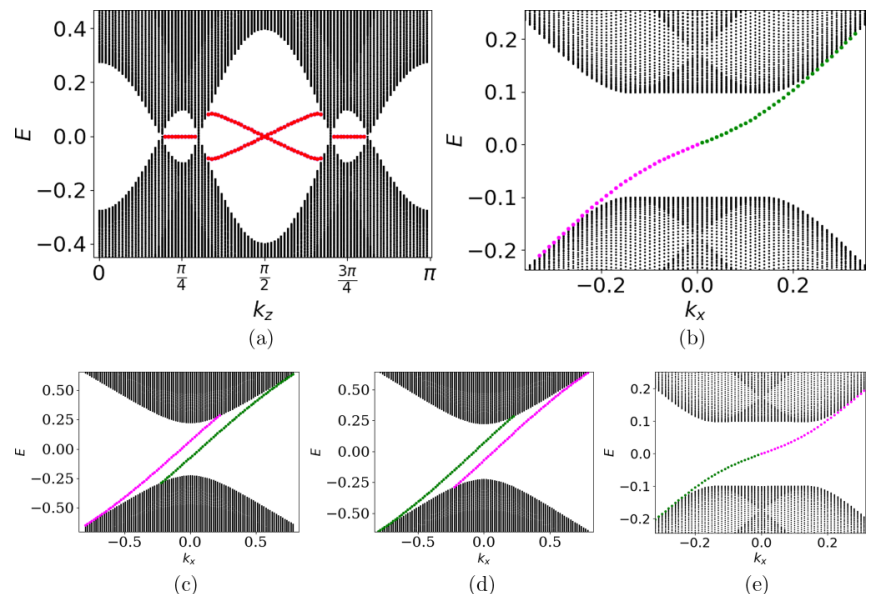

FIG. 2. The bulk and surface spectra for the topological CDW ordering with emergent Weyl nodes of Eq. (6). The open boundaries are perpendicular to the $y$ axis, and only surface states localized at the $y=0$ boundary are shown. Parameter values are $\rho / t=0.1$ and $V_{0} / t=0.2$. (a) The dispersions along the cut at $k_{x}=0$ with varying $k_{z}$ in the reduced $\mathrm{BZ}$ with $0 \leqslant k_{z} \leqslant \pi . \mathbf{K}_{e}^{-}$and $\mathbf{K}_{h}^{+}$at $k_{z}<0$ are folded into the reduced BZ. Surface state spectra are plotted in red. The dispersions for varying $k_{x}$ are shown for constant $k_{z}$ cuts at (b) $k_{z}=\frac{\pi}{4}$, (c) $k_{z}=\frac{3 \pi}{8}$, (d) $k_{z}=\frac{5 \pi}{8}$, and (e) $k_{z}=\frac{3 \pi}{4}$. Green and magenta respectively indicate surface states with majority weight in the $0 \leqslant k_{z} \leqslant \pi$ and $-\pi \leqslant k_{z} \leqslant 0$ components.

helical basis and thus needs to be treated more carefully. Taking into account the $4 \pi$ flux from the Dirac string penetrating the south pole, or equivalently changing the gauge choice to place the singularity at the north pole, this Weyl node can also be shown to possess positive chirality as well.

The positive chiralities of $\mathbf{K}_{n, s}^{+}$are in fact determined by the chiralities of the original band-structure Weyl nodes $\mathbf{K}_{e, h}^{+}$, which are away from the chemical potential and hence lie in the high-energy sector. Nevertheless, they still determine the chirality of the low-energy Weyl nodes, independent of the details of the mechanism of the CDW ordering. Typically, the low-energy physics is not sensitive to the details at high energy, but the topological structure at low energy in our case is inherited from the topology at high energy, and thus the emergence of the low-energy Weyl fermions are topologically protected.

Similar analysis can also be performed in parallel for the CDW ordering connecting the nested Fermi surfaces $\mathrm{FS}_{h,-1}$ and $\mathrm{FS}_{e, 1}$ surrounding $\mathbf{K}_{h, e}^{-}$, respectively. The two low-energy Weyl nodes denoted $\mathbf{K}_{n, s}^{-}$on the nested $\mathrm{FS}_{h,-1}$ and $\mathrm{FS}_{e, 1}$ have negative chirality, which is again determined by the Weyl nodes $\mathbf{K}_{h, e}^{-}$at high energies. In total, the sum of chiralities of all the Weyl nodes, including the original band structure ones and the interaction-induced ones, remain zero as required by the Nielsen-Ninomiya theorem [52,53].

The emergent Weyl nodes and novel topological surface states are demonstrated in the quasiparticle energy spectra in Fig. 2, where we take open boundary conditions along the $y$ direction and periodic boundary conditions along the $x$ and $z$ directions. Because of the nesting vector $\mathbf{Q}=(0,0, \pi)$, the reduced Brillouin zone (BZ) with $0 \leqslant k_{z} \leqslant \pi$ is considered. As shown in Fig. 2(a), two pairs of emergent zero-energy 
Weyl nodes $\mathbf{K}_{s, n}^{-}$and $\mathbf{K}_{s, n}^{+}$appear at the $k_{z}$ axis near $\mathbf{K}_{h}^{-}$and $\mathbf{K}_{e}^{+}$in the bulk quasiparticle energy spectrum. The surface states localized at the $y=0$ boundary are shown in color. Away from the Fermi surface, there are two branches of chiral surface states for $\left(\mathbf{K}_{n}^{-}\right)_{z}<k_{z}<\left(\mathbf{K}_{s}^{+}\right)_{z}$, due to BZ folding of the original Fermi arcs in Fig. 1(b). The number of branches of surface states changes as $k_{z}$ moves across $\mathbf{K}_{s}^{-}, \mathbf{K}_{n}^{-}, \mathbf{K}_{s}^{+}$, and $\mathbf{K}_{n}^{+}$, as shown in Figs. 2(b)-2(e). The surface states inside the CDW gaps with $\left(\mathbf{K}_{s}^{ \pm}\right)_{z}<k_{z}<\left(\mathbf{K}_{n}^{ \pm}\right)_{z}$ are quasiparticles that are superpositions of electron states at $\mathbf{k}$ and $\mathbf{k}+\mathbf{Q}$ due to the particle-hole pairing near the Fermi surface. The component with majority weight changes when these surface states cross zero energy in Figs. 2(b) and 2(e), in contrast to the surface states shown in Figs. 2(c) and 2(d) where each branch of surface states is dominated by a single component. Thus, the CDW gaps admit zero-energy quasiparticles in equal superposition of $\mathbf{k}$ and $\mathbf{k}+\mathbf{Q}$ states that are a CDW analog of Majorana modes.

These zero-energy boundary modes are protected by symmetry and topology. In the basis $\Psi(\mathbf{k})=$ $\left(c_{A}(\mathbf{k}), c_{B}(\mathbf{k}), c_{A}(\mathbf{k}+\mathbf{Q}), c_{B}(\mathbf{k}+\mathbf{Q})\right)^{T}$, the Hamiltonian takes the form $H=\sum_{\mathbf{k}} \Psi^{\dagger}(\mathbf{k}) H(\mathbf{k}) \Psi(\mathbf{k})$, where $H(\mathbf{k})=$ $I_{\Sigma} \otimes h(\mathbf{k})+\Sigma_{z} \otimes V(\mathbf{k})+\Sigma_{x} \otimes \rho(\mathbf{k}), \quad$ and $\quad I_{\Sigma} \quad$ and $\quad \Sigma_{i}$ are, respectively, the identity and Pauli matrices acting on the $(\mathbf{k}, \mathbf{k}+\mathbf{Q})$ space, the CDW analog of Nambu space. The Hamiltonian $H(\mathbf{k})$ possesses the symmetry $\mathcal{C} H\left(k_{x}, k_{y}, k_{z}\right) \mathcal{C}^{-1}=-H\left(-k_{x},-k_{y}, k_{z}\right)$, where $\mathcal{C}$ is an antiunitary operator given by $\mathcal{C}=\Sigma_{x} \otimes \tau_{x} K$ and $K$ is the complex conjugation operator. Therefore, each momentum slice with fixed $k_{z}$ not at the emergent CDW gap nodes can be regarded as a two-dimensional gapped system belonging to the $D$ symmetry class [54], which admits a $\mathbb{Z}$ classification characterized by the Chern number, and the number of chiral boundary modes crossing zero energy is precisely the Chern number. This is also evident from the projected Hamiltonian Eq. (7): Each momentum slice at fixed $k_{z}$ describes an odd-parity pairing state. Inside the FS, $t|p|<\mu$, the two-dimensional low-energy effective Hamiltonian is in the weak-pairing state and hosts a topologically protected zero-energy mode [55].

A natural question is whether the projection to the lowenergy helical Fermi surface remains valid as the CDW ordering strength $\rho$ becomes large. We find that the zero-energy Weyl nodes remain robust until $\rho$ reaches a critical value $\rho_{c}$. As $\rho$ is increased, the low-energy Weyl nodes $\mathbf{K}_{n}^{-}$and $\mathbf{K}_{s}^{+}$are pushed closer to each other, as are $\mathbf{K}_{s}^{-}$and $\mathbf{K}_{n}^{+}$. As shown in Fig. 3(a), $\mathbf{K}_{s}^{-}$and $\mathbf{K}_{n}^{+}$first merge at $k_{z}=0$, opening a gap, and $\mathbf{K}_{n}^{-}$and $\mathbf{K}_{s}^{+}$remain separated. Further enlarging $\rho$, $\mathbf{K}_{n}^{-}$and $\mathbf{K}_{s}^{+}$merge next at $k_{z}=\frac{\pi}{2}$ after which the gap opens. After both pairs of zero-energy Weyl nodes merge, the system becomes fully gapped, as shown in Fig. 3(b). The surface states in this case cross the bulk gap as $k_{x}$ is varied.

Increasing $\rho$ beyond the point at which the system first becomes gapped, there must eventually be an additional gap closing followed by a gap opening. To see this, consider that in the $\rho \rightarrow \infty$ limit, the spectrum must be concentrated close to $\pm \rho$, the eigenvalues of $H_{\rho}$. In this large- $\rho$ limit, there can then be no surface states crossing the gap, and the surface states shown in Fig. 3(b) must eventually disappear following a second transition as $\rho$ increases beyond $\rho=0.6 t$. A more
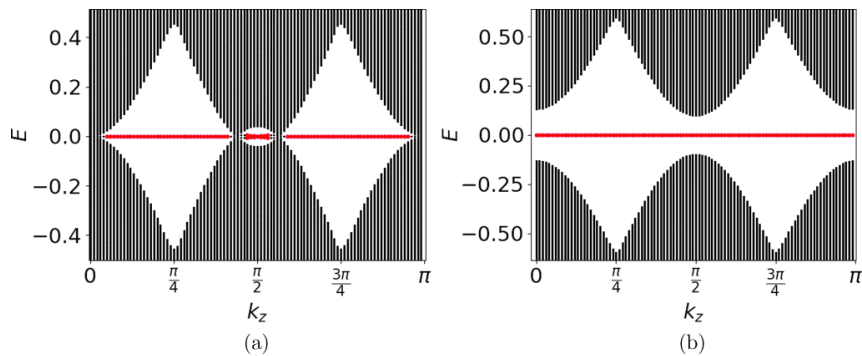

FIG. 3. Evolution of the low-energy Weyl nodes as the ordering strength $\rho$ is increased with fixed $V=0.2 t$. (a) At $\rho \approx 0.458 t$, a gap at $k_{z}=0$ starts to open as $\mathbf{K}_{s}^{-}$merges with $\mathbf{K}_{n}^{+}$. (b) By $\rho=0.6 t$, there is a gap at all $k_{z}$ after $\mathbf{K}_{n}^{-}$has merged with $\mathbf{K}_{s}^{-}$at $k_{z}=\frac{\pi}{2}$.

detailed explanation is presented in SM II [50]. This process is similar to the transition between weak- and strong-coupling topological superconductivity, in which the strong-coupling state is topologically trivial.

Discussion. The general requirement for material realizations of monopole CDW states is the presence of nested Fermi surfaces enclosing Weyl points of the same chirality, which can occur in either an inversion-breaking or, as in this work, a time-reversal-breaking Weyl semimetal. In addition to the intrinsic ordering mechanism, CDW states can also be driven by an ultrafast laser pulse, as demonstrated in a layered chalcogenide system [56]. If the CDW state instead occurs between Fermi surfaces enclosing Weyl points of opposite chiralities, the gap function has zero Berry phase and can either be fully gapped or nodal. In such a case, the gap function symmetry is represented by conventional spherical harmonics or their lattice counterpart and is determined energetically by specific ordering mechanisms.

To experimentally characterize the monopole CDW, phase sensitive bulk detection, which can be provided by resonant inelastic x-ray scattering (RIXS), is desired to extract the nontrivial total vorticity of phase winding in the gap function. It has been shown that the RIXS difference spectrum for crosspolarized $\mathrm{x}$ rays exhibits zero crossings as an unambiguous indicator of the topological winding numbers of a Weyl Hamiltonian [57]. Hence, current high-energy-resolution RIXS is further expected to probe the topological excitations near the emergent Weyl nodes where the CDW-Weyl Hamiltonians exhibit nontrivial phase windings in the monopole CDW ordering. Furthermore, RIXS can map out the nontrivial total vorticity of the monopole CDW gap function, which is absent in the usual gapped or even nodal CDW states. Detailed analysis will be deferred to a future publication.

It would be interesting to investigate orderings besides the monopole CDW in this system. Since most Weyl semimetals are not strongly correlated, we consider another type of Fermi surface instability - superconductivity. In this model, the superconductivity arising from zero-momentum pairing occurs between Fermi surfaces with opposite Chern numbers and is thus an example of the recently proposed monopole superconductivity [48]. The superconducting gap function is also nodal and characterized by monopole harmonics. It is possible to only have the CDW instability without superconductivity or, if their energy scales are comparable, to 
have both instabilities coexist. Analogous to the coexistence of the CDW and superconductivity seen in the transitionmetal dichalcogenide $\mathrm{NbSe}_{2}$ [58-62], competition between monopole harmonic orders is possible, which will be studied in future work.

Conclusions. We have studied the inter-Fermi-surface particle-hole pairing between well-nested Fermi surfaces enclosing Weyl nodes of the same chirality. The CDW ordering in this case gives rise to gap functions possessing topologically protected nodes independent of the details of ordering interactions. These nodes manifest in the bulk singleparticle energy spectra as zero-energy Weyl nodes, which allows the topology of the surface Fermi arcs to change. The nodal structure corresponds to a topological CDW state whose order parameter carries monopole symmetries. As the ordering strength increases to the strong-coupling regime, the zero energy Weyl nodes merge and disappear, and the system becomes fully gapped.

Acknowledgments. We thank Y. Wang, P. Abbamonte, C. Broholm, S. Kourtis, and T. McQueen for helpful discussion. This work was supported by NSF CAREER Grant No. DMR-1848349 and in part by the Alfred P. Sloan Research Fellowships.

E.B. and C.S. contributed equally to this work.
[1] G. Grüner, Rev. Mod. Phys. 60, 1129 (1988).

[2] A. J. Heeger, S. Kivelson, J. R. Schrieffer, and W. P. Su, Rev. Mod. Phys. 60, 781 (1988).

[3] R. E. Thorne, Phys. Today 49(5), 42 (1996).

[4] W. P. Su, J. R. Schrieffer, and A. J. Heeger, Phys. Rev. B 22, 2099 (1980).

[5] C. Nayak, Phys. Rev. B 62, 4880 (2000).

[6] K. Fujita, M. H. Hamidian, S. D. Edkins, C. K. Kim, Y. Kohsaka, M. Azuma, M. Takano, H. Takagi, H. Eisaki, S.-i. Uchida, A. Allais, M. J. Lawler, E.-A. Kim, S. Sachdev, and J. C. Séamus Davis, Proc. Natl. Acad. Sci. USA 111, E3026 (2014).

[7] E. Fradkin, S. A. Kivelson, and J. M. Tranquada, Rev. Mod. Phys. 87, 457 (2015).

[8] F. D. M. Haldane, Phys. Rev. Lett. 61, 2015 (1988).

[9] C.-Z. Chang, J. Zhang, X. Feng, J. Shen, Z. Zhang, M. Guo, K. Li, Y. Ou, P. Wei, L.-L. Wang, Z.-Q. Ji, Y. Feng, S. Ji, X. Chen, J. Jia, X. Dai, Z. Fang, S.-C. Zhang, K. He, Y. Wang et al., Science 340, 167 (2013).

[10] C. L. Kane and E. J. Mele, Phys. Rev. Lett. 95, 226801 (2005).

[11] C. L. Kane and E. J. Mele, Phys. Rev. Lett. 95, 146802 (2005).

[12] B. A. Bernevig and S.-C. Zhang, Phys. Rev. Lett. 96, 106802 (2006).

[13] B. A. Bernevig, T. L. Hughes, and S.-C. Zhang, Science 314, 1757 (2006).

[14] S. Murakami, New J. Phys. 9, 356 (2007).

[15] X. Wan, A. M. Turner, A. Vishwanath, and S. Y. Savrasov, Phys. Rev. B 83, 205101 (2011).

[16] A. A. Burkov and L. Balents, Phys. Rev. Lett. 107, 127205 (2011).

[17] K.-Y. Yang, Y.-M. Lu, and Y. Ran, Phys. Rev. B 84, 075129 (2011).

[18] G. Xu, H. Weng, Z. Wang, X. Dai, and Z. Fang, Phys. Rev. Lett. 107, 186806 (2011).

[19] T. Meng and L. Balents, Phys. Rev. B 86, 054504 (2012).

[20] W. Witczak-Krempa and Y. B. Kim, Phys. Rev. B 85, 045124 (2012).

[21] G. Y. Cho, J. H. Bardarson, Y.-M. Lu, and J. E. Moore, Phys. Rev. B 86, 214514 (2012).

[22] P. Hosur, Phys. Rev. B 86, 195102 (2012).

[23] C. Fang, M. J. Gilbert, X. Dai, and B. A. Bernevig, Phys. Rev. Lett. 108, 266802 (2012).

[24] G. B. Halász and L. Balents, Phys. Rev. B 85, 035103 (2012).
[25] D. T. Son and B. Z. Spivak, Phys. Rev. B 88, 104412 (2013).

[26] P. Hosur and X. Qi, C. R. Phys. 14, 857 (2013).

[27] Z. Wang and S.-C. Zhang, Phys. Rev. B 87, 161107(R) (2013).

[28] M. M. Vazifeh and M. Franz, Phys. Rev. Lett. 111, 027201 (2013).

[29] R. Nandkishore, D. A. Huse, and S. L. Sondhi, Phys. Rev. B 89, 245110 (2014)

[30] P. Hosur, X. Dai, Z. Fang, and X.-L. Qi, Phys. Rev. B 90, 045130 (2014).

[31] A. C. Potter, I. Kimchi, and A. Vishwanath, Nat. Commun. 5, 5161 (2014).

[32] F. D. M. Haldane, arXiv:1401.0529.

[33] H. Wei, S.-P. Chao, and V. Aji, Phys. Rev. B 89, 014506 (2014).

[34] S. A. Yang, H. Pan, and F. Zhang, Phys. Rev. Lett. 113, 046401 (2014).

[35] H. Weng, C. Fang, Z. Fang, B. A. Bernevig, and X. Dai, Phys. Rev. X 5, 011029 (2015).

[36] A. A. Burkov, J. Phys.: Condens. Matter 27, 113201 (2015).

[37] S. Borisenko, D. Evtushinsky, Q. Gibson, A. Yaresko, K. Koepernik, T. Kim, M. Ali, J. van den Brink, M. Hoesch, A. Fedorov, E. Haubold, Y. Kushnirenko, I. Soldatov, R. Schäfer, and R. J. Cava, Nat. Commun. 10, 3424 (2019).

[38] J. Xiong, S. K. Kushwaha, T. Liang, J. W. Krizan, M. Hirschberger, W. Wang, R. Cava, and N. Ong, Science 350, 413 (2015).

[39] S.-Y. Xu, N. Alidoust, I. Belopolski, Z. Yuan, G. Bian, T.-R. Chang, H. Zheng, V. N. Strocov, D. S. Sanchez, G. Chang, C. Zhang, D. Mou, Y. Wu, L. Huang, C.-C. Lee, S.-M. Huang, B. Wang, A. Bansil, H.-T. Jeng, T. Neupert et al., Nat. Phys. 11, 748 (2015).

[40] S.-Y. Xu, I. Belopolski, N. Alidoust, M. Neupane, G. Bian, C. Zhang, R. Sankar, G. Chang, Z. Yuan, C.-C. Lee, S.-M. Huang, H. Zheng, J. Ma, D. S. Sanchez, B. Wang, A. Bansil, F. Chou, P. P. Shibayev, H. Lin, S. Jia, and M. Z. Hasan, Science 349, 613 (2015).

[41] S.-M. Huang, S.-Y. Xu, I. Belopolski, C.-C. Lee, G. Chang, B. Wang, N. Alidoust, G. Bian, M. Neupane, C. Zhang et al., Nat. Commun. 6, 7373 (2015).

[42] B. Q. Lv, H. M. Weng, B. B. Fu, X. P. Wang, H. Miao, J. Ma, P. Richard, X. C. Huang, L. X. Zhao, G. F. Chen, Z. Fang, X. Dai, T. Qian, and H. Ding, Phys. Rev. X 5, 031013 (2015).

[43] B. Lu, K. Yada, M. Sato, and Y. Tanaka, Phys. Rev. Lett. 114, 096804 (2015). 
[44] N. Xu, H. M. Weng, B. Q. Lv, C. E. Matt, J. Park, F. Bisti, V. N. Strocov, D. Gawryluk, E. Pomjakushina, K. Conder et al., Nat. Commun. 7, 11006 (2016).

[45] B. Yan and C. Felser, Ann. Rev. Condens. Matter Phys. 8, 337 (2017).

[46] B. Bradlyn, L. Elcoro, J. Cano, M. Vergniory, Z. Wang, C. Felser, M. Aroyo, and B. A. Bernevig, Nature (London) 547, 298 (2017).

[47] N. P. Armitage, E. J. Mele, and A. Vishwanath, Rev. Mod. Phys. 90, 015001 (2018).

[48] Y. Li and F. D. M. Haldane, Phys. Rev. Lett. 120, 067003 (2018).

[49] T. T. Wu and C. N. Yang, Nucl. Phys. B 107, 365 (1976).

[50] See Supplemental Material at http://link.aps.org/supplemental/ 10.1103/PhysRevResearch.2.012078 for a lattice model of the Weyl semimetal system with nesting and analysis of the charge density wave gap-closing transitions in the strong-coupling regime.
[51] Y. Wang and P. Ye, Phys. Rev. B 94, 075115 (2016).

[52] H. B. Nielsen and M. Ninomiya, Nucl. Phys. B 185, 20 (1981).

[53] H. B. Nielsen and M. Ninomiya, Nucl. Phys. B 193, 173 (1981).

[54] S. Ryu, A. Schnyder, A. Furusaki, and A. Ludwig, New J. Phys. 12, 065010 (2010).

[55] N. Read and D. Green, Phys. Rev. B 61, 10267 (2000).

[56] K. Sun, S. Sun, C. Zhu, H. Tian, H. Yang, and J. Li, Sci. Adv. 4, eaas9660 (2018).

[57] S. Kourtis, Phys. Rev. B 94, 125132 (2016).

[58] K. Takita and K. Masuda, J. Low Temp. Phys. 58, 127 (1985).

[59] T. Yokoya, T. Kiss, A. Chainani, S. Shin, M. Nohara, and H. Takagi, Science 294, 2518 (2001).

[60] X. Xi, L. Zhao, Z. Wang, H. Berger, L. Forró, J. Shan, and K. F. Mak, Nat. Nanotechnol. 10, 765 (2015).

[61] C.-S. Lian, C. Si, and W. Duan, Nano Lett. 18, 2924 (2018).

[62] F. Zheng and J. Feng, Phys. Rev. B 99, 161119(R) (2019). 